Research report

\title{
Environmental factors and teenagers' personalities: The role of personal and familial Socio-Cultural Level
}

\author{
Elisa Menardo ${ }^{\mathrm{a}}$, Giulia Balboni $^{\mathrm{b}, *}{ }^{*}$ Roberto Cubelli ${ }^{\mathrm{c}}$ \\ a University of Verona, Lungadige Porta Vittoria, 17, 37129, Verona, Italy \\ b University of Perugia, Piazza G. Ermini, 1, 06123, Perugia, Italy \\ c University of Trento, Corso Bettini, 31, 38068, Rovereto, Italy
}

\section{H I G H L I G H T S}

- Socio-Cultural Level indicators are associated with different personality profiles.

- Relationship between Socio-Cultural Level and teen personality is sex specific.

- Personal more than familial SocioCultural Level is associated with teen personality.

- Parents' Socio-Cultural Levels could influence children's personalities indirectly.

\section{A R T I C L E I N F O}

\section{Article history:}

Received 2 August 2016

Received in revised form 20 February 2017

Accepted 22 February 2017

Available online 24 February 2017

\section{Keywords:}

Big Five

Socio-Cultural Level

Cultural Capital

Social Capital

Socioeconomic Status

Environment
G R A P H I C A L A B S T R A C T

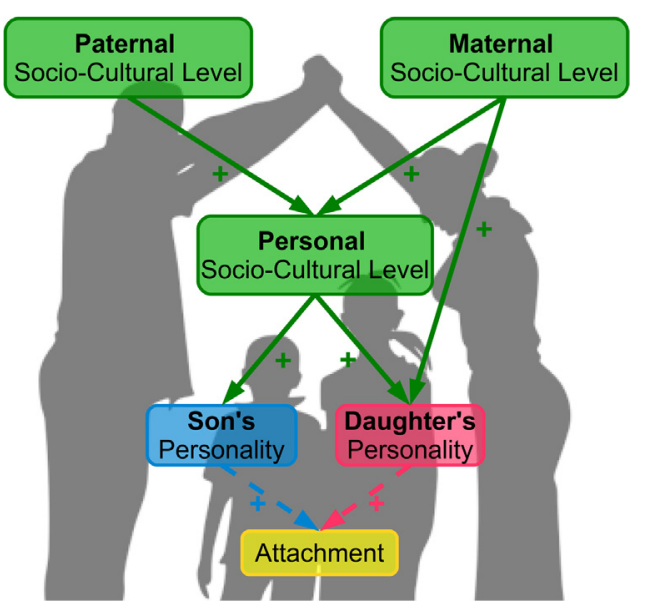

\begin{abstract}
A B S T R A C T
Environmental (e.g., socio-cultural context), individual (e.g., genetic makeup), and interpersonal (e.g., caregiver-children relationships) factors can play a crucial role in shaping the development of the teenagers' personality. In this study, we focused on the Socio-Cultural Level that designates the set of preferences, knowledge, and behaviors that characterize an individual's way of life and depend on his or her cultural, social, and economic resources. We studied the relationship between Socio-Cultural Level (personal, maternal, and paternal) and Big Five personality traits of 191 teenagers living in the same geographical area. Results showed that Socioeconomic Status (i.e., parental education level and occupational prestige), which is the only dimension generally measured in investigations on Socio-Cultural Level, was not related with personality. In contrast, Cultural Capital and Social Capital were associated with different personality traits. Personal Cultural Capital was related to Openness to experience of boys and girls and to Extraversion of girls; personal Social Capital was related to Extraversion of girls, Emotional stability of boys, and Agreeableness of both boys and girls; maternal Cultural Capital was associated with Openness to experience of daughters. Overall, the personality of teenagers was more related to their own
\end{abstract}

\footnotetext{
* Corresponding author at: Department of Philosophy, Social and Human Sciences and Education, University of Perugia, Piazza G. Ermini, 1, 06123, Perugia, Italy. E-mail addresses: elisa.menardo@univr.it (E. Menardo), giulia.balboni@unipg.it(G. Balboni), roberto.cubelli@unitn.it (R. Cubelli).
} 
Cultural and Social Capital than to the Cultural and Social Capital of their parents. Moreover, the relationship between Cultural Capital and Social Capital of boys/girls and of fathers/mothers was moderate in strength. It seems that parents influence the development of personality of their teenagers indirectly, their Socio-Cultural Level shaping the Socio-Cultural Level of their sons and daughters.

(C) 2017 Elsevier B.V. All rights reserved.

\section{Introduction}

Personality refers to the individual differences in ways of thinking, behaving and interacting; it reflects stable and consistent qualities and traits resulting from early interactions with parents and caregivers. One of the most widespread and well-validated models of personality is the Big Five model [1], which comprises five dimensions. Extraversion reflects a keen interest in people and events, and energy for exploring the world; Agreeableness denotes the ability to interact with others and to be sympathetic, kind, and affectionate; Conscientiousness refers to responsibility and reliability; Emotional stability (frequently discussed in its reversed form, Neuroticism) is a measure of emotional resilience; Openness to experience expresses independence and intellectual curiosity.

Over the years, psychologists have identified several factors affecting the development of personality: individual (e.g., biological and genetic substrates), interpersonal (e.g., mother-child relationships), and environmental (e.g., socio-economic conditions) factors. One of the most influential environmental factors is the Socio-Cultural Level [2], which is strictly related to the enduring reciprocal interactions between the individual and his/her immediate environment, defined as proximal processes by Bronfenbrenner $[3,4]$.

According to Lamont and Lareau [5], Socio-Cultural Level designates the set of preferences, knowledge, and behaviors that characterize the way of life and depend on the cultural, social, and economic resources of an individual or of the adults in a family. Socio-Cultural Level is a multidimensional construct and includes three different components [6,7]: Socioeconomic Status (SES), Cultural Capital (CC), and Social Capital (SC).

SES denotes the position of a person or of a family within a social system where values such as professional prestige, educational level, economic resources, power, and access to information are not evenly distributed [8]. Typically, investigations of SES consider income, educational level, and professional prestige [9]. CC, first defined by Bourdieu and Passeron [10], refers to knowledge and use of the cultural codes that are relevant in the community where an individual lives [5]. Because CC is a highly context-dependent construct, researchers generally use ad hoc questionnaires based on the characteristics of the target population, investigating artistic, recreational and associative interests, and activities. SC refers to the resources associated with social network connections [11] linking people within groups (bonding capital) or across groups (bridging capital). Despite different theoretical views [6,7], the assessment of SC typically considers the extent to which the individual's connections appear to be durable, trustworthy, and able to offer socioeconomic resources [11,12].

Over the years, psychological studies have shown a positive correlation between SES and the Big Five traits in adults [12-15], with different indicators of SES showing different results. Whereas educational level [15] and occupational prestige [12] appear to be associated with all the dimensions of personality, income showed significant associations with Extraversion, Conscientiousness, and Emotional stability, but not with Openness to experience or Agreeableness [15]. Although rarely investigated, CC and SC also have shown a relationship to personality in adults. Both have shown associations with Extraversion, Emotional stability, and Openness to experience; SC has also been associated with Conscientiousness [16].

Only Pellicci, Menardo, Balboni, and Cubelli [17] have explored the three dimensions of Socio-Cultural Level together to study their relationship to personality in adults. They found different patterns of relationship according to the participant's sex: women's personalities appeared to be more related to CC, whereas men's personalities seemed more strongly tied to education level.

As the association between Socio-Cultural Level and personality has been studied only in adulthood, the purpose of the present study was to explore it in teenagers. In particular, we aimed to verify whether teenagers with different Socio-Cultural Levels show different Big Five profiles. We took into account all the dimensions of Socio-Cultural Level at both personal and familial (maternal and paternal) levels. We hypothesized different profiles of personality in boys and girls according to personal and familial Socio-Cultural Level. As regards personal Socio-Cultural Level, based on previous studies on adulthood [17], we hypothesized that girls' personality should be more associated to CC, whereas boys' personality should be more associated to the educational level. For what concerns familial Socio-Cultural Level, given that paternal and maternal SES showed different association with children's personality [15], we hypothesized different associations with teenagers' personality also for paternal and maternal CC and SC. Moreover, since paternal education level appears to have more influence on children's Big Five traits than maternal education level [15], we hypothesized different profiles of sons' and daughters' personality according to maternal and paternal Socio-Cultural Levels.

Finally, previous studies have found a moderate influence of familial Socio-Cultural Level on personal Socio-Cultural Level [18]. Therefore, it is possible that, at least at some degree, familial Socio-Cultural Level could have an indirect influence on teenagers' personality, by shaping their Socio-Cultural Level. To this aim, we studied the correlation between personal CC and SC of boys and girls and personal CC and SC of their mothers and fathers. We predicted moderate to high correlation coefficients.

This study is particularly useful because, to our knowledge, it is the first time that the influence of environmental factors on teenagers' personality has been investigated, taking into account the different personal and familial dimensions of Socio-Cultural Level and considering sex of teenagers and parents as moderator variables. This study aimed to understand the interplay between an individual biological dimension (i.e., sex) and the socio-cultural context in the development of the Big Five traits.

\section{Material and methods}

\subsection{Participants}

One hundred ninety-one teenagers, attending six different high schools (three general education and three pre-university education) in the metropolitan area of Lucca (Italy), participated in the study. Of the 500 students contacted, 223 (45\%) agreed to take part in the survey. Of these, 32 were excluded: 30 did not complete all the questionnaires and 2 exceeded the cut-off for simulation on 
Table 1

Characteristics of teenagers, mothers, and fathers.

\begin{tabular}{lccc}
\hline & $\begin{array}{c}\text { Teenager } \\
(n=191)\end{array}$ & $\begin{array}{c}\text { Mother } \\
(n=150)\end{array}$ & $\begin{array}{c}\text { Father } \\
(n=125)\end{array}$ \\
\hline $\begin{array}{l}\text { Sex }(\%) \\
\text { F-M }\end{array}$ & $116(61)-75(39)$ & - & - \\
Age & & & \\
$\quad M(S D)$ & $16.1(1.6)$ & $46.2(4.7)$ & $48.4(5.1)$ \\
range & $13-20$ & $30-58$ & $35-63$ \\
Educational level (\%) & & & \\
$\quad$ Low & $90(47)$ & $60(40)$ & $69(53)$ \\
$\quad$ High & $101(53)$ & $90(60)$ & $61(47)$ \\
Occupational prestige (\%) & & & \\
$\quad$ Low & - & $49(33)$ & $46(35)$ \\
$\quad \begin{array}{l}\text { Medium } \\
\text { High }\end{array}$ & - & $48(32)$ & $38(29)$ \\
\end{tabular}

either the Lie Scale of the Big-Five Questionnaire-2 [19] or the social desirability scale Balanced Inventory of Desirable Responding- 6 Short Form (BIDR-6 Short Form) [20].

Among the 191 teenagers, 153 also returned questionnaires from their mothers, whereas 130 returned questionnaires from fathers. Three mothers and five fathers were detected as simulators and therefore excluded. Table 1 shows the characteristics of the groups of teenagers, mothers, and fathers. Informed consent was obtained for all participants, and no monetary incentive was given.

\subsection{Instruments}

\subsubsection{Personality}

To measure personality, we used the Big-Five Questionnaire2 (BFQ-2), containing 134 items, each rated on a 5-point Likert scale [19]. Each item addresses one of five main traits (Extraversion, Agreeableness, Conscientiousness, Emotional stability, and Openness to experience) or the Lie Scale. All BFQ-2 scales showed good internal consistency (Cronbach's $\alpha$ ranging from 0.79 to 0.90 ). For each scale, the standard score $(M=50 ; S D=10)$ was computed following the Italian standardization norms [19].

\subsubsection{Cultural Capital}

CC was measured by the Scale of Cultural Capital [21]. The scale comprises 20 multiple-choice items, each rated on a 5-point Likert scale, to estimate cultural interests and activities: reading books, using foreign languages, visiting museums, attending cultural events, or being a member of cultural, social, political, religious and recreational groups. Cronbach's $\alpha$ for this scale has been reported at $0.80[21]$.

\subsubsection{Social Capital}

The Personal Social Capital Scale comprises 10 composite items with a total of 54 sub-items [11]. It allows measurement of bonding and bridging capitals. ${ }^{1}$ Chinese [11] and English [22] versions of the scale have shown excellent internal consistency and factorial structure. Cronbach's $\alpha$ for this scale has been reported at 0.87 [11].

\footnotetext{
1 Examples of items assessing SC (1) for bonding capital and (2) for bridging capital:. (1) With how many of people in each of the following categories do you keep a routine contact? (a) Your family members. (b) Your relatives. (c) People in your neighborhood. (d) Your friends. (e) Your co-workers/fellows; and (f) Your country fellows/old classmates. (2) In each of the two types of groups/organizations, how many will help you upon your request? (a) Governmental, political, economic and social groups/organizations (political parties, women's groups, village committees, trade unions, cooperate associations, volunteer groups, etc.). (b) Cultural, recreational, and leisure groups/organizations (religious, country fellows, alumni, sport, music, dances, crafts, games, etc.).
}

In the present study, an Italian adaptation with excellent content validity was used [23].

\subsubsection{Socioeconomic Satus}

SES was measured through occupational prestige and educational level. Occupational prestige was assessed with the Italian Occupational Prestige Scale, an ordinal scale made up of 110 occupational categories ordered according to the prestige associated with each of them [24]. We developed a questionnaire (Scale of Employment) to collect all the information required (type of job, kind of employment contract, and field of work) to identify the corresponding category on the Italian Occupational Prestige Scale.

\subsubsection{Social Desiderability}

To detect attempts at simulation, we used the Lie Scale of the BFQ-2 and the BIDR-6 Short Form. The Lie Scale of the BFQ-2 comprises 14 items rated on a 5-point Likert scale. Individuals were considered simulators when the obtained standard score was equal to or above 66 (i.e., 1.5 standard deviations above the mean) [19]. The 16-item BIDR-6 Short Form uses a 6-point Likert scale to evaluate the unconscious tendency to provide honest but positively-biased responses, as well as the habitual and conscious presentation of a favorable public image [20]. Individuals were considered simulators when their total score exceeded the 95th centile [20]. This scale has shown adequate Cronbach's $\alpha$ [20] and good factorial structure.

\subsection{Procedure}

Teenagers received three booklets that contained all relevant questionnaires: one booklet to be completed by themselves and two to be completed by their mothers and fathers separately. Each booklet comprised the BFQ-2, the Scale of Cultural Capital, the Personal Social Capital Scale, and the BIDR-6 Short Form. Parents also received the Scale of Employment. To avoid any effect of the instruments' order of presentation, booklets were prepared according to 4 balanced orders for the teenagers, 12 balanced orders for mothers and 12 balanced orders for fathers. The single exception to the balancing was that the BIDR- 6 Short Form was always given at the end.

The teenagers were classified according to each indicator of Socio-Cultural Level to enable investigation of their different profiles. Scores for personal, maternal, and paternal CC and SC, and maternal and paternal occupational prestige were categorized into three sub-groups (low, medium, and high) based on the 33rd and 66th centile of the distribution on the corresponding scales. For personal education level (see Table 1), teenagers were coded as either low (first or second high school grade) or high (third, fourth, or fifth high school grade) based on the Italian high school curriculum. The low group included 46 teenagers in the first grade $(M$ age $=14.3$ years [ $S D=0.67$ ], range $=13-17)$ and 44 in the second grade $(M$ age $=15.4$ years $[S D=0.54]$, range $=15-17)$. The high comprised 28 teenagers in the third grade $(M$ age $=16.3$ years $[S D=0.44]$, range $=16-17), 46$ in the fourth grade $(M$ age $=17.2$ years $[S D=0.72]$, range $=15-19)$, and 27 in the fifth grade $(M$ age $=18.7$ years $[S D=0.71]$, range $=17-20)$. Maternal and paternal educational levels were also classified into two subgroups: low (vocational certificate or lower) and high (high school or higher). The subgroups of teenagers obtained for each Socio-Cultural Level indicator did not differ on age or sex.

\subsection{Data analysis}

As a preliminary analysis, we checked for normal distribution and for the presence of univariate outliers according to Tabachnick and Fidell's suggestion [25]. Teenagers who obtained a score more 
Table 2

Means $(S D)$ of BFQ-2 scale standard score for which ANOVA revealed statistically significant differences between boys with different Socio-Cultural Levels.

\begin{tabular}{|c|c|c|c|c|c|}
\hline & \multicolumn{3}{|c|}{ Socio-Cultural Level } & \multicolumn{2}{|c|}{ ANOVA } \\
\hline & $\begin{array}{c}\text { Low } \\
M(S D)\end{array}$ & $\begin{array}{l}\text { Medium } \\
M(S D)\end{array}$ & $\begin{array}{l}\text { High } \\
M(S D)\end{array}$ & $F\left(\omega^{2}\right)$ & Post- hoc $(d)$ \\
\hline & \multicolumn{3}{|c|}{ Personal Cultural Capital ${ }^{\mathrm{a}}$} & & \\
\hline & $n=27$ & $n=28$ & $n=20$ & & \\
\hline \multirow[t]{3}{*}{ Openness to experience } & $\begin{array}{l}39.52 \\
(6.45)\end{array}$ & $\begin{array}{l}46.39 \\
(8.49)\end{array}$ & $\begin{array}{l}46.60 \\
(9.41)\end{array}$ & $\begin{array}{l}5.59^{* *} \\
(0.05)\end{array}$ & $\begin{array}{l}\mathrm{L}<\mathrm{M}^{*}(0.93) \\
\mathrm{L}<\mathrm{H}^{*}(0.92)\end{array}$ \\
\hline & \multicolumn{3}{|c|}{ Personal Social Capital $^{\mathrm{a}}$} & & \\
\hline & $n=24$ & $n=25$ & $n=26$ & & \\
\hline \multirow{4}{*}{ Emotional stability } & $\begin{array}{c}44.54 \\
(11.13)\end{array}$ & $\begin{array}{l}46.88 \\
(7.68)\end{array}$ & $\begin{array}{c}53.62 \\
(10.06)\end{array}$ & $\begin{array}{l}5.22^{* *} \\
(0.04)\end{array}$ & $\mathrm{L}<\mathrm{H}^{* *}(0.88)$ \\
\hline & $\begin{array}{l}41.83 \\
(7.72)\end{array}$ & $\begin{array}{l}48.00 \\
(9.07)\end{array}$ & $\begin{array}{l}48.96 \\
(9.70)\end{array}$ & $\begin{array}{l}4.27^{*} \\
(0.03)\end{array}$ & $\mathrm{L}<\mathrm{H}^{*}(0.81)$ \\
\hline & \multicolumn{3}{|c|}{ Paternal Cultural Capital ${ }^{\mathrm{b}}$} & & \\
\hline & $n=13$ & $n=18$ & $n=15$ & & \\
\hline Conscientiousness & $\begin{array}{l}51.08 \\
(6.49)\end{array}$ & $\begin{array}{l}43.11 \\
(6.77)\end{array}$ & $\begin{array}{c}50.27 \\
(11.13)\end{array}$ & $\begin{array}{l}6.3^{* *} \\
(0.07)\end{array}$ & n.s. \\
\hline
\end{tabular}

Note. ${ }^{\mathrm{a}} d f=2,182 .{ }^{\mathrm{b}} d f=2,117 .{ }^{*} p<0.05 .{ }^{* *} p<0.01 .{ }^{* * *} p<0.001$.

Table 3

Means (SD) of BFQ-2 scale standard score for which ANOVA revealed statistically significant differences between girls with different Socio-Cultural Levels.

\begin{tabular}{|c|c|c|c|c|c|}
\hline & \multicolumn{3}{|c|}{ Socio-Cultural Level } & \multicolumn{2}{|c|}{ ANOVA } \\
\hline & $\begin{array}{c}\text { Low } \\
M(S D)\end{array}$ & $\begin{array}{c}\text { Medium } \\
M(S D)\end{array}$ & $\begin{array}{l}\text { High } \\
M(S D)\end{array}$ & $F\left(\omega^{2}\right)$ & Post- hoc $(d)$ \\
\hline & \multicolumn{3}{|c|}{ Personal Cultural Capital ${ }^{\mathrm{a}}$} & & \\
\hline & $n=29$ & $n=39$ & $n=45$ & & \\
\hline Extraversion & $\begin{array}{l}43.65 \\
(9.07)\end{array}$ & $\begin{array}{c}48.66 \\
(11.47)\end{array}$ & $\begin{array}{c}50.22 \\
(10.00)\end{array}$ & $\begin{array}{l}3.92^{*} \\
(0.03)\end{array}$ & $\mathrm{L}<\mathrm{H}^{*}(0.68)$ \\
\hline \multirow[t]{4}{*}{ Openness to experience } & 40.86 & 44.03 & 50.20 & $11.35^{* * *}$ & $\begin{array}{l}\mathrm{L}<\mathrm{H}^{* * *}(1.14) \\
\mathrm{M}<\mathrm{H}^{* *}(0.68)\end{array}$ \\
\hline & \multirow{2}{*}{\multicolumn{3}{|c|}{ Personal Social Capital ${ }^{\mathrm{a}}$}} & & \\
\hline & & & & & \\
\hline & $n=33$ & $n=39$ & $n=41$ & & \\
\hline Extraversion & $\begin{array}{l}45.55 \\
(9.57)\end{array}$ & $\begin{array}{c}46.67 \\
(10.09)\end{array}$ & $\begin{array}{c}51.29 \\
(11.15)\end{array}$ & $\begin{array}{l}3.62^{*} \\
(.03)\end{array}$ & $\mathrm{L}<\mathrm{H}^{*}(0.56)$ \\
\hline \multirow[t]{3}{*}{ Agreeableness } & $\begin{array}{c}44.85 \\
(10.78)\end{array}$ & $\begin{array}{c}50.85 \\
(10.69)\end{array}$ & $\begin{array}{c}49.10 \\
(10.86)\end{array}$ & $\begin{array}{l}3.11^{*} \\
(.02)\end{array}$ & $\mathrm{L}<\mathrm{M}^{*}(0.57)$ \\
\hline & \multicolumn{3}{|c|}{ Maternal Cultural Capital ${ }^{\mathrm{b}}$} & & \\
\hline & $n=30$ & $n=28$ & $n=34$ & & \\
\hline Openness to experience & $\begin{array}{l}41.80 \\
(9.58)\end{array}$ & $\begin{array}{l}44.43 \\
(9.43)\end{array}$ & $\begin{array}{l}49.88 \\
(9.33)\end{array}$ & $\begin{array}{l}6.3^{* *} \\
(0.07)\end{array}$ & $\mathrm{L}<\mathrm{H}^{* *}(0.87)$ \\
\hline
\end{tabular}

Note. ${ }^{\text {a }} d f=2,182 .{ }^{\mathrm{b}} d f=2,141 .{ }^{*} p<0.05 .{ }^{* *} p<0.01 .{ }^{* * *} p<0.001$.

than 3.29 standard deviations over or under the corresponding subgroup mean in at least one BFQ-2 scale were excluded from the corresponding comparison. We also investigated the presence of multivariate outliers.

We conducted a series of two-way between-subjects multivariate analyses of variance (MANOVAs): three for personal Socio-Cultural Level and four for each of maternal and paternal Socio-Cultural Level. Sex was the first independent variable and each of the Socio-Cultural Level indicators (educational level, CC, CS, or occupational prestige for maternal and paternal SocioCultural Level only) was added one at a time as the second independent variable. The standard scores on the BFQ-2 scales were entered as dependent variables. To locate the sources of the global differences reflected by the MANOVAs, we evaluated the effects of each independent variable on each BFQ-2 scale with a series of ANOVAs followed by Bonferroni's post hoc comparisons.
In cases of statistically significant differences, we computed partial $\eta^{2}\left(\eta_{p}^{2}\right)$ for multivariate analysis, $\omega^{2}$ for univariate analysis, and Cohen's $d$ for post-hoc analysis $[25,26]$. In agreement with Cohen's criteria [26], effect sizes were evaluated as negligible $\left(\eta^{2}\right.$, $\left.\omega^{2}<0.01 ; d<0.20\right)$, small $\left(0.01 \leq \eta^{2}\right.$, $\left.\omega^{2}<0.06 ; 0.20 \leq d<0.50\right)$, medium $\left(0.06 \leq \eta^{2}\right.$ p, $\left.\omega^{2}<0.14,0.50 \leq d<0.80\right)$, or large $\left(\eta^{2}, \omega^{2} \geq\right.$ $0.14, d \geq 0.80)$.

\section{Results}

3.1. Big Five personality profile of boys and girls associated with personal, maternal and paternal Socio-Cultural Level

MANOVA revealed that personal CC had a multivariate effect (Wilk's $\lambda=0.827, F(10,356)=3.56, p<0.001, \eta^{2}=0.09$ ) and a univariate effect on Openness to experience $(F(2182)=12.76$, 
Table 4

Pearson correlation coefficents between personal Cultural Capital and Social Capital of boys and girls and those of their mother and father.

\begin{tabular}{|c|c|c|}
\hline & \multicolumn{2}{|c|}{ Teenagers' Personal Socio-Cultural Level } \\
\hline & Cultural Capital & Social Capital \\
\hline \multicolumn{3}{|l|}{ Boys } \\
\hline \multicolumn{3}{|l|}{ Maternal $(n=55)$} \\
\hline Cultural Capital & 0.298 & - \\
\hline Social Capital & - & 0.322 \\
\hline \multicolumn{3}{|l|}{ Paternal $(n=46)$} \\
\hline Cultural Capital & 0.304 & - \\
\hline Social Capital & - & 0.332 \\
\hline \multicolumn{3}{|l|}{ Girls } \\
\hline \multicolumn{3}{|l|}{ Maternal $(n=95)$} \\
\hline Cultural Capital & 0.484 & - \\
\hline Social Capital & - & 0.405 \\
\hline \multicolumn{3}{|l|}{ Paternal $(n=79)$} \\
\hline Cultural Capital & 0.364 & - \\
\hline Social Capital & - & 0.422 \\
\hline
\end{tabular}

Note. For all the coefficients, $p<0.05$.

$\left.p<0.001, \omega^{2}=0.11\right)$. Also, personal SC had a multivariate effect (Wilks' $\lambda=0.838, F(10,356)=3.28, p<0.001, \eta^{2} p=0.08$ ) and univariate effects $(p<0.01)$ on Extraversion $\left(F(2,182)=5.48, \omega^{2}=0.05\right)$, Agreeableness $\left(F(2,182)=6.19, \omega^{2}=0.05\right)$, and Emotional stability $\left(F(2,182)=6.20, \omega^{2}=0.05\right)$. As for familial Socio-Cultural Level, MANOVA revealed only a univariate effect of paternal occupational prestige on Extraversion $\left(F(2,117)=3.74, p<0.05, \omega^{2}=0.04\right)$.

MANOVA revealed as significant only the interaction of Sex X paternal CC on Conscientiousness, but the effect size was small $\left(F(2,117)=3.52, p<0.05, \omega^{2}<0.04\right)$. In contrast, personal CC and SC showed different multivariate effects $(p<0.05)$ for boys and girls. CC was linked to different patterns of personality traits only in girls (Wilk's $\lambda=0.825, F(10,356)=3.59, \eta^{2} \mathrm{p}=0.09$ ), whereas SC had effects for both girls (Wilk's $\lambda=0.902, F(10,356)=1.88, \eta^{2}{ }_{p}=0.05$ ) and boys (Wilk's $\lambda=0.888, F(10,356)=2.18, \eta^{2}$ p $=0.06$ ).

Subsequent ANOVAs revealed the dimensions of personality involved. As illustrated in Tables 2 and 3, teenagers exhibited different personality profiles depending on Socio-Cultural Level dimensions of personal, maternal and paternal CC, and personal SC. Personal CC was related to different scores on Openness to experience in boys and girls and on Extraversion in girls only. Maternal CC was related to different scores on Openness to experience in girls only. Paternal CC showed a main effect on Conscientiousness in boys only, but post-hoc comparisons found no statistically significant differences. Personal SC was related to Agreeableness scores in boys and girls, to Extraversion in girls, and to Emotional stability in boys. For all these comparisons, teenagers with higher SocioCultural Levels had higher scores on BFQ-2 scales. Effect sizes were always at least moderate. No differences in personality profiles were related to personal, maternal or paternal education levels, or to maternal or paternal occupational prestige, or SC.

\subsection{Relationship between Cultural Capital and Social Capital of boys and girls and those of their mothers and fathers}

We also computed Pearson's correlation coefficients between CC and SC of boys/girls and those of their fathers/mothers. We subsequently compared, for both boys and girls, the magnitude of the correlation coefficients obtained (a) between personal CC and maternal (or paternal) CC with those obtained between personal SC and maternal (or paternal) SC; (b) between personal CC (or CS) and maternal CC (or CS) with those obtained between personal CC (or CS) and paternal CC (or CS). For this aim, we used the correlation comparison strategies for dependent, non-overlapping correlations and for independent samples, respectively $[27,28]$. Finally, we com- pared the correlation coefficients obtained for the boys with the corresponding coefficients obtained for the girls. To this end, we used the correlation comparison strategy for independent samples [27].

As can be seen in Table 4, the coefficients were moderate. No statistically significant differences emerged in the correlation comparisons. $^{2}$

\section{Discussion}

This study aimed to ascertain whether personal, maternal, and paternal dimensions of Socio-Cultural Level are related to the personality profiles of boys and girls. We found evidence of different relationships, thus highlighting the multidimensional nature of Socio-Cultural Level.

Our results appear to be at variance with those on adulthood reported in the literature [15-17]. First of all, whereas the personal education level in adulthood seems to be related to all personality traits [15], in teenagers it appears to be associated with no traits. This difference could be due to the small number of high school grades, which could flatten the variability. Alternatively, it could be due to the fact that teenagers have not yet completed their formal education.

Second, personal CC has different relationships with the personality profiles of adults and teenagers. In adults, CC has been found to be related to Extraversion, Emotional stability, Openness to experience [16], and Conscientiousness [17]; our study showed that in teenagers, CC is related to Openness to experience and, in girls only, to Extraversion. All studies converge in showing a relationship between CC and Openness to experience. We hypothesize that, independently of age and sex, CC can promote a positive attitude to novelty and other cultures and can stimulate creativity, desire for variety and interest in new knowledge. However, this study involved a single time point, and there is no way to know the direction of effects. Therefore, it is equally plausible that teenagers with open personalities seek more out cultural activities and participation, and teenagers with more extraverted personalities may accumulate more SC. Longitudinal studies are necessary to explore deeply the direction of the effect in this relationship.

Finally, whereas in adults personal SC is related to almost all personality traits [16,17], in teenagers we found a relationship with Agreeableness in both boys and girls, with Emotional stability in boys only, and with Extraversion in girls only. It is possible that the reciprocal interactions between SC - and with Socio-Cultural Level in general - and personality occur continuously across the lifespan and therefore are more clearly noticeable only in adulthood. By definition, teenagers' personalities and Socio-Cultural Levels are changing and unstable; consequently, the relationship between Socio-Cultural Level and personality could be less conspicuous.

\footnotetext{
2 As suggested by two reviewers, we also used regression analysis to investigate whether personal, maternal, and paternal Socio-Cultural Level indicators predict teenagers' Big Five traits. We run standard multiple regression analyses for boys and for girls separately. Predictors were CC and CS of personal, maternal, and paternal Socio-Cultural Level in addition with occupational prestige of maternal and paternal Socio-Cultural Level. Observed variables were each of the Big Five traits one at a time. We found that personal CC predicted Openness to experience of both boys and girls; personal CS predicted Emotional stability of both boys and girls and Agreeableness of boys only. Maternal CC predicted Openness to experience of daughters. Finally, paternal CC, CS, and occupational prestige predicted Agreeableness of sons (see analyses in the Supplementary material). Overall, these results are in agreement with what found using MANOVA. Only for paternal Socio-Cultural Level of boys, regression found associations between all Socio-Cultural Level indicators and Agreeableness which were not found with MANOVA. However, the number of boys with available paternal Socio-Cultural Level was very small, thus limiting the power of the analyses. Further investigations should address this issue.
} 
As regards familial Socio-Cultural Level, we found that the only relevant relationship was that of maternal CC with their daughters' Openness to experience. Since girls and boys who grow up in families with a high CC tend to reproduce the cultural activities of their parents [18], it is possible that girls, more than boys, share activities and experiences with their mothers. However, as maternal CC has the same strength of relationship with CC of daughters and of sons, the link between maternal CC and Openness to experience in daughters only cannot be due to a stronger relationship between maternal CC and daughters' CC. On the contrary, maternal CC seems to promote directly intellectual curiosity, creativity and a preference for novelty and variety more in daughters than in sons.

In investigating the relationship between CC and SC of boys/girls and of fathers/mothers, we found moderate Pearson correlation coefficients. Therefore, it is possible that because parents' SocioCultural Levels shape the Socio-Cultural Levels of their teenagers, this indirectly influences, at least to some degree, the development of the teenagers' personalities. Correlation comparisons revealed that the maternal and paternal CC and SC equally correlate with those of boys and girls, suggesting a similar intergenerational transmission at the social and cultural levels.

This study has inevitable limitations. We categorized CC, SC, and occupational prestige measures into three categories to enable use of MANOVAs. In this way, we were able to investigate the differences in personality profiles according to each dimension of personal and familial Socio-Cultural Level. However, the sample-dependent cut-points make for difficult comparisons across studies. Other statistical techniques, like regression analysis, would have allowed using continuous variables but would have produced a shift in the research aims, leading to study how much each Socio-Cultural Level dimension predicts the Big Five traits. In the present study, we aimed to investigate which Big Five personality profiles are associated with each Socio-Cultural Level dimension.

Our findings showed that sex of teenagers and parents moderates the relationship between teenagers' personality and personal, maternal, and paternal Socio-Cultural Level. These results are in agreement with the bioecological model proposed by Bronfenbrenner $[4,29]$. According to this model, the development of personality results from the reciprocal interaction between the individual's own biology and the ecological system, which is composed of nested socially organized subsystems. The Microsystem refers to the settings in which an individual has direct social interactions (e.g., family, school, groups of peers, workplace, and neighborhood). The connections between two or more Microsystems give rise to the Mesosystem (e.g., the relationships between parents and teachers) or to the Exosystem which includes at least one Microsystem having an indirectly influence on the individual (e.g., the connection between home and parents' workplace). The Macrosystem designates the actual culture of the individual and comprises belief systems, bodies of knowledge, socioeconomic status, life style, and opportunity structures. Within the Bronfenbrenner's model, the Socio-Cultural Level could be assumed as a measure of both the Macro- and Micro-system of an individual.

We also found SES (parental educational level and occupational prestige), which is usually the only measure used in investigations on Socio-Cultural Level, is not related with personality. It seems that, at least in teenagers, SES is not an appropriate indicator to study how individual and his/her environment interact in determining the development of personality.

Finally, we found that teenagers' personalities are more related to personal than to paternal or maternal Socio-Cultural Levels. It is plausible that personal Socio-Cultural Level plays a decisive role in a stage of life during which youngsters feel the need to differentiate their own identity and to distinguish themselves from parents. Teenagers are searching for autonomy; their personal interests, knowledge and relationships, which are often independent of those of their parents, could influence their personalities more than does parental bonding. In contrast, in an earlier stage of development, the interests and experiences of children evolve within those of the family. We infer that in children, the relationship between familial Socio-Cultural Level and personality should be more evident than in teenagers. Further studies should investigate this relationship in children to determine whether a different pattern of results emerges.

Over the years, psychological studies have shown evidence of SES as one of the factors influencing adults' personality [12-15]; in contrast, CC and SC have been measured and investigated only rarely. Therefore, our results are particularly useful given that we have investigated both personal and familial dimensions of Socio-Cultural Level with both sexes of teenagers and parents as moderator variables.

Our results have implications also for the study of adults' attachment. Personality and attachment are strongly related. According to Bowlby [30,31], Attachment Theory can be seen as a theory of the development of the personality over a lifetime. Interactions between child and caregiver, from the early months and years of life, determine the maturation of a particular attachment style. Different behaviors towards himself/herself and the others reveal different styles based on different mental structures and representations. The capacity to conceptualize the mental states of oneself and others has been theorized as the core feature of interpersonal functioning and personality development [32].

It has been found that Emotional stability is strongly related to marital satisfaction [33], whereas Extraversion and Agreeableness are positively related to attachment security and negatively related to attachment avoidance [e.g.,34,35]. Based on our results, Emotional stability, Extraversion, and Agreeableness are all related to personal SC of the teenager. Therefore, we can surmise that, at least to some degree, personal SC is related to attachment style. It is plausible that teenagers with a large social network and strong social supports develop a more secure attachment style than do those with limited SC. In turn, teenagers with a secure attachment style may be more able than those with anxious or avoidant styles to take advantage of social relationships.

According to Bronfenbrenner [3], the effect of mother-child interactions on personality development is more powerful than that of environmental context. Investigating the influence of personal and familial Socio-Cultural Level can provide important information to our understanding of personality development and the interplay between interpersonal and socio-environmental factors.

Neuroscientific research on personality supports a biologicallybased explanatory model of the Big Five. Studies have shown associations between Big Five traits - in particular, Extraversion, Agreeableness, Emotional stability, and Conscientiousness - and different brain regions [36]. Interestingly, there is no evidence of an association between the volume of defined brain areas and Openness to experience, which is the trait with the strongest relationship to Socio-Cultural Level.

We are aware of the interaction between genetic and environmental factors in determining attachment and personality [e.g.,37,38]. The environmental factors may have a unilateral impact on the individual's development or may be incidental and negligible accidental and passively perceived. However, as recently suggested by Kikusui and Hiroi [39], environmental factors can also be self-generated and actively sought. Consequently, the amount and quality of social interactions and opportunities may be the effect of genetic factors and individual predispositions. The measurement of Socio-Cultural Level and the study of the direct and indirect influences of familial SCL on teenagers' personalities may 
provide useful information for investigating these two types of environmental factors.

\subsection{Conclusions}

The main novel contribution of this study is the investigation of the relationship between teenagers' personalities and all dimensions of personal and familial Socio-Cultural Level, taking sex of teenagers and parents as moderator variables. Future longitudinal studies, using both attachment and personality measures, will shed more light on the complex processes linking attachment, personality, and personal and familial Socio-Cultural Level with quality of relationships in adulthood.

\section{Founding}

This research did not receive any specific grant from funding agencies in the public, commercial, or not-for-profit sectors.

\section{Ethical standards}

This study was performed in accordance with the ethical standards laid down in the 2013 version of the Declaration of Helsinki. Informed consent was obtained from parents or other caregivers, as appropriate.

\section{Conflict of interest}

The authors declare that they have no conflict of interest.

\section{Appendix A. Supplementary data}

Supplementary data associated with this article can be found, in the online version, at http://dx.doi.org/10.1016/j.bbr.2017.02.038.

\section{References}

[1] R.R. McCrae, P.T. Costa Jr, F. Ostendorf, A. Angleitner, M. Hrebícková, M.D Avia, J. Sanz, M.L. Sánchez-Bernardos, M.E. Kusdil, R. Woodfield, P.R. Saunders, P.B. Smith, Nature over nurture: temperament, personality, and life span development, J. Pers. Soc. Psychol. 78 (2000) 173-186.

[2] A. Coscarelli, G. Balboni, R. Cubelli, The problem of measuring the socio-cultural level in psychological research, in: T.E. Scruggs, M.A Mastropieri (Eds.), Advances in Learning and Behavioral Disabilities, Volume 20: International perspectives, Elsevier Science Ltd, Oxford, 2007, pp. $163-180$.

[3] U. Bronfenbrenner, Ecological models of human development, in: L.T. Husten, T.N. Postlehwaite (Eds.), International Encyclopedia of Education, second ed., Pergamon Press, Oxford, 1994, pp. 3-27.

[4] U. Bronfenbrenner, G.W. Evans, Developmental science in the 21st century: emerging questions, theoretical models, research designs and empirical findings, Soc. Dev. 9 (2000) 115-125.

[5] M. Lamont, A. Lareau, Cultural capital: allusions, gaps and glissandos in recent theoretical developments, Sociol. Theory 6 (1988) 153-168.

[6] P. Bourdieu, Le capital social [The social capital], actes rech, Sci. Soc. 31 (1980) $2-3$.

[7] J.S. Coleman, Social capital in the creation of human capital, Am. J. Sociol. 94 (1988) S95-S120.

[8] M.H. Bornstein, R.H. Bradley, Socioeconomic Status, Parenting, and Child Development, Lawrence Erlbaum Associates, Mahwah New Jersey, 2003.

[9] R.D. Conger, M.B. Donnellan, An interactionist perspective on the socioeconomic context of human development, Annu. Rev. Psychol. 58 (2007) 175-199.

[10] P. Bourdieu, J.C. Passeron, La Reproduction [The Reproduction], Editions de Minuit, Paris (1970); trad. it. La Riproduzione, Guaraldi, Rimini, 1972.

[11] X. Chen, B. Stanton, J. Gong, X. Fang, X. Li, Personal Social Capital Scale: an instrument for health and behavioral researchers, Health Educ. Res. 24 (2009) 306-317.
[12] H. Cheng, A. Furnham, The association between parental socio-economic conditions childhood intelligence, adult personality traits, social status and mental well-being, Soc. Indic. Res. 117 (2014) 653-664.

[13] R.I. Damian, R. Su, M. Shanahan, U. Trautwein, B.W. Roberts, Can personality traits and intelligence compensate for background disadvantage? Predicting status attainment in adulthood, J. Pers. Soc. Psychol. 109 (2015) 473-489.

[14] B.P. Chapman, K. Fiscella, I. Kawachi, R. Duberstein, Personality socioeconomic status, and all-Cause Mortality in the United States, Am. J. Epidemiol. 171 (2009) 83-92

[15] C.R. Jonassaint, I.C. Siegler, J.C. Barefoot, C.L. Edwards, B.W. Redford, Low life course socioeconomic status (SES) is associated with negative NEO PI-R personality patterns, Int. J. Behav. Med. 11 (2011) 13-21.

[16] E. Khodadady, R. Zabihi, School performance cultural, social and personality factors and their relationships with majoring in foreign and first languages, ELT J. 4 (2011) 63-73.

[17] F. Pellici, E. Menardo, G. Balboni, R. Cubelli, Effetti del livello socio-culturale sulle dimensioni di personalitalEffects of Socio-Cultural Level on personality dimensions], Giorn. It. Psicol. 4 (2015) 875-884, http://dx.doi.org/10.1421/ 81946.

[18] J. de Vries, P.M. de Graaf, Is the intergenerational transmission of high cultural activities biased by the retrospective measurement of parental high cultural activities? Soc. Indic. Res. 85 (2008) 311-327.

[19] G.V. Caprara, C. Barbaranelli, L. Borgogni, M. Vecchione, Big-Five Questionnaire-2, Giunti O.S. Organizzazioni Speciali, Firenze, 2007.

[20] A. Bobbio, A.M. Manganelli, Measuring social desirability responding. A short version of Paulhus' BIDR 6, TPM Test, Psychom. Methodol. Appl. Psychol. 18 (2011) 117-135

[21] A. Coscarelli, G. Balboni, R. Cubelli, Scala di Capitale Culturale [Scale of Cultural Capital] (2011) [Manuscript not pubblished].

[22] A.J. Archuleta, C.R. Miller, Validity evidence for the translated version of the Personal Social Capital Scale among people of Mexican descent, J. Soc. Social Work Res. 2 (2011) 39-53.

[23] G. Balboni, A. Coscarelli, A.M. Magnani, R. Cubelli, Scala di Capitale Sociale Personale [Personal Social Capital Scale] (2007) [Manuscript not pubblished].

[24] C. Meraviglia, L. Accornero, La valutazione sociale delle occupazioni nell'Italia contemporanea: Una nuova scala per vecchie ipotesi [The social evaluation of occupations in contemporary Italy: a new scale for old cases], Quad. Sociol. 45 (2007) 19-73.

[25] B.G. Tabachnick, L.S. Fidell, Using Multivariate Statistics, 6th ed., Harper Collins, New York, 2013.

[26] J. Cohen, Statistical Power Analysis for the Behavioral Sciences, 2nd ed., Erlbaum, Hillsdale, N.J, 1988.

[27] J. Cohen, P. Cohen, Applied Multiple Regression/correlation Analysis for the Behavioral Science, second ed., Erlbaum, Hillsdale, New York, 1983.

[28] T.E. Raghunathan, R. Rosenthal, D.B. Rubin, Comparing correlated but nonoverlapping correlations, Psychol. Methods 1 (1996) 178-183.

[29] U. Bronfenbrenner, S.J. Ceci, Nature-nurture reconceptualized in developmental perspective: a bioecological model, Psychol. Rev. 101 (1994) 568-586.

[30] J. Bowlby, Attachment and Loss, Vol. 1: Attachment, Basic Books, New York, 1969.

[31] M.D.S. Ainsworth, J. Bowlby, An ethological approach to personality development, Am. Psychol. 46 (1991) 333-341.

[32] P. Fonagy, M. Target, Mentalization and personality disorder in children: a current perspective from the Anna Freud Centre, in: T. Lubbe (Ed.), The Borderline Psychotic Child: A Selective Integration, Taylor \& Francis, Philadelphia, 2000, pp. 69-89.

[33] D. Heller, D. Watson, R. Ilies, The role of person versus situation in life satisfaction: a critical examination, Psychol. Bull. 130 (2004) 574-600.

[34] E.E. Noftle, P.R. Shaver, Attachment dimensions and the big five personality traits: associations and comparative ability to predict relationship quality, J. Res. Pers. 40 (2006) 179-208.

[35] D. Watson, B. Hubbard, D. Wiese, General traits of personality and affectivity as predictors of satisfaction in intimate relationships: evidence from self- and partner-ratings, J. Pers. 68 (2000) 413-449.

[36] C.G. DeYoung, J.B. Hirsh, M.S. Shane, X. Papademetris, N. Rajeevan, J.R. Gray, Testing predictions from personality neuroscience: brain structure and the Big Five, Psychol. Sci. 21 (2010) 820-828.

[37] J.L. Borelli, P.A. Smiley, H.F. Rasmussen, A. Gómez, L.C. Seaman, E.L. Nurmi, Interactive effects of attachment and FKBP5 genotype on school-aged children's emotion regulation and depressive symptoms, Behav. Brain Res. (2016), http://dx.doi.org/10.1016/j.bbr.2016.07.035.

[38] G. Esposito, A. Truzzi, P. Setoh, D.L. Putnick, K. Shinohara, M.H. Bornstein, Genetic predispositions and parental bonding interact to shape adults' physiological responses to social distress, Behav. Brain Res. (2016), http://dx doi.org/10.1016/j.bbr.2016.06.042.

[39] T. Kikusui, N. Hiroi, A self-generated environmental factor as a potential contributor to atypical early social communication in autism, Neuropsychopharmacology (2017), http://dx.doi.org/10.1038/npp.2016.225. 